\title{
RNF183 promotes proliferation and metastasis of colorectal cancer cells via activation of NF- $\kappa$ B-IL-8 axis
}

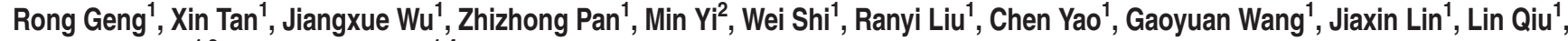 \\ Wenlin Huang ${ }^{\star, 1,3}$ and Shuai Chen ${ }^{*, 1,4}$
}

Colorectal cancer (CRC) is one of the most common malignant tumors worldwide, which is a heterogeneous disease and main risk factors are associated with inflammation, family history, genetic mutations, epigenetics, and so on. Ring finger domain proteins have been reported involved in carcinogenesis, whereas their roles in CRC are rarely studied. Here, we reanalyzed the expression of 202 RNF family members in CRC using published microarray data from GEO database and found that RNF183 is markedly upregulated in tumor tissues. RNF183 high expression is significantly associated with tumor size $(P=0.012)$, tumor invasive depth $(P=0.004)$, TNM stage $(P=0.01)$, and distant metastasis $(P=0.009)$. CRC patients with high expression of RNF183 have poor overall survival $(P<0.001)$ and progression-free survival $(P<0.001)$. Functional studies suggest that RNF183 facilitates growth, migration, and invasion of CRC cells in vitro and promotes tumor proliferation and metastasis in vivo. Mechanistically, RNF183 activates NF- $\kappa$ B signal pathway through P65 and stimulates the transcription of multifunctional chemokine IL-8. Blockage of NF- $\kappa$ B by small molecule inhibitor or depletion of IL-8 by siRNA attenuates the function of RNF183 to promote cell migration. Moreover, the regulation of RNF183 on IL-8 transcription and cell viability/motility is dependent on its E3 ubiquitin ligase activity. Our study provided proof of principle to show that RNF183 promotes proliferation and metastasis of CRC cells via activation of NF- $\kappa$ B-IL-8 axis.

Cell Death and Disease (2017) 8, e2994; doi:10.1038/cddis.2017.400; published online 10 August 2017

Colorectal cancer (CRC) is the third most common malignancy of cancer morbidity worldwide. ${ }^{1}$ The overall neoplastic lethality rate of $\mathrm{CRC}$ is $\sim 50 \%$. $^{1}$ The mechanisms underlying CRC is complex and many key pathways and genes are involved, including $A P C, W n t, \beta$-catenin, EGFR/RAS/RAF/MEK, TFG- $\beta$, and so on. ${ }^{2,3}$ Although targeted drugs such as anti-EGFR monoclonal antibodies cetuximab and panitumumab have been use for the treatment of metastatic colorectal cancer (mCRC), $<20 \%$ of mCRC patients with wild-type KRAS benefit from these drugs when they were used as monotherapy. ${ }^{4,5}$ On the other hand, targeting downstream kinases of EGFR, such as BRAF and MEK, shows less efficacy in CRC therapy. ${ }^{4,6}$ Thus, it is critical to discover new mechanisms that drive the initiation and development of CRC to provide new targets for therapy.

The RING finger (RNF) protein family is a complex set of proteins containing a RNF domain, which typically includes 40-60 amino acids. ${ }^{7}$ More than 200 RNF family genes have been identified, and many of which exhibit wide range of functions in different biological and pathological processes. ${ }^{8}$ Many RNF family members have been reported to play key roles in carcinogenesis and development. For instance, RNF31 is a subunit of linear ubiquitin chain assembly complex (LUBAC) and is affected by germline polymorphisms among activated B-cell-like subtype of diffuse large B-cell lymphoma patients $(\sim 7.8 \%)$, which increase LUBAC enzymatic activity thus in turn activate NF- $\kappa$ B and promote tumorigenesis. ${ }^{9}$ Highfrequent mutation of RNF43 is observed in gastrointestinal cancer including CRCs and cystic pancreatic tumors, which sensitize tumor cells to Wnt inhibitors. ${ }^{10,11}$ Identification of more RNF family members associated with cancer, including colorectal carcinoma, will help to understand the process of carcinogenesis and to develop new therapeutic strategies.

In the current study, we reanalyzed GEO data set GSE8671 and compared the expression of 202 RNF family genes between colorectal adenoma and adjacent normal tissues. Our analyses revealed significant increased expression of RNF183 in tumor tissues. Although RNF183 is found upregulated in inflammatory bowel disease (IBD), a disease highly associated with $\mathrm{CRC}$, and activates classic NF- $\kappa \mathrm{B}$ pathway, ${ }^{12-14}$ its role in cancer cells remains largely unknown. Here, we report that RNF183 is an independent prognostic factor in CRC patients, and high RNF183 level is closely related to tumor size, invasive depth, TNM stage and distant metastasis. RNF183 upregulates the expression of multifunction chemokine IL-8 through NF- $\kappa$ B pathway and promotes cancer cell growth, migration, invasion and metastasis in vitro and in vivo. Therefore, our study indicates that RNF183 is a CRC associated gene and could serve as a target for the development of new therapeutic strategies.

\footnotetext{
${ }^{1}$ Sun Yat-sen University Cancer Center, State Key Laboratory of Oncology in South China, Collaborative Innovation Center of Cancer Medicine, Guangzhou, P.R. China; ${ }^{2}$ School of Food Science and Engineering, Yangzhou University, Yangzhou, P.R. China; ${ }^{3}$ Guangdong Provincial Key Laboratory of Tumor Targeted Drugs and Guangzhou Enterprise Key Laboratory of Gene Medicine, Guangzhou Doublle Bioproducts Co. Ltd., Guangzhou, P.R. China and ${ }^{4}$ Guangdong Esophageal Cancer Institute, Sun Yat-sen University Cancer Center, Guangzhou, P.R. China

*Corresponding author: S Chen or W Huang, Sun Yat-sen University Cancer Center, State Key Laboratory of Oncology in South China, Collaborative Innovation Center of Cancer Medicine, 651 East Dongfeng Road, Guangzhou 510060, P.R. China. Tel: +86 20 87342303; Fax: +86 2087343146 ; E-mail: shuaichen2010@ @otmail.com or hwenl@mail.sysu.edu.cn

Received 14.3.17; revised 18.6.17; accepted 04.7.17; Edited by M Daugaard
} 

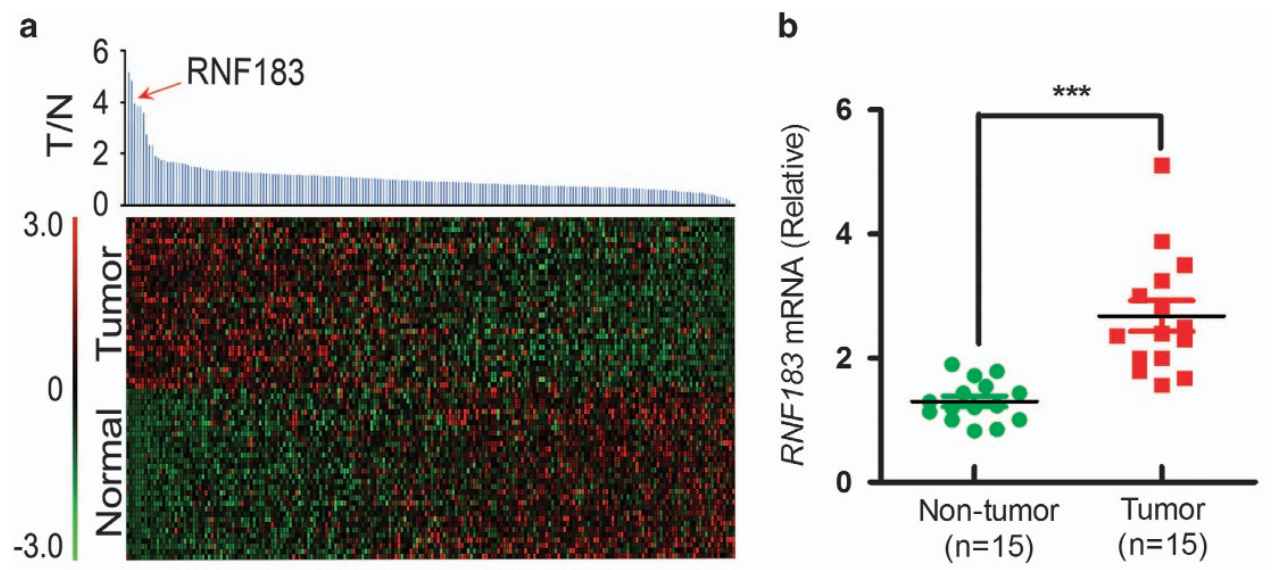

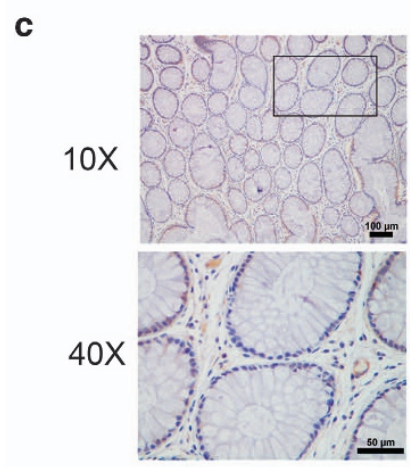

Adjacent normal mucosal tissue

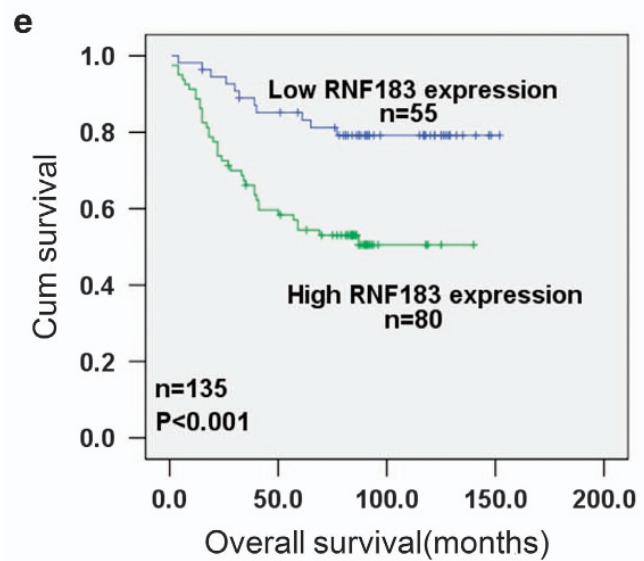

d

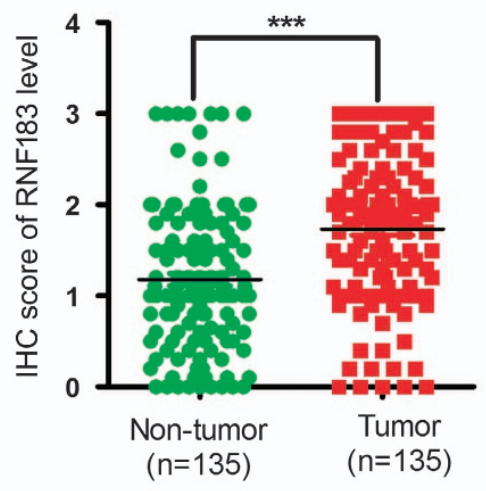

f

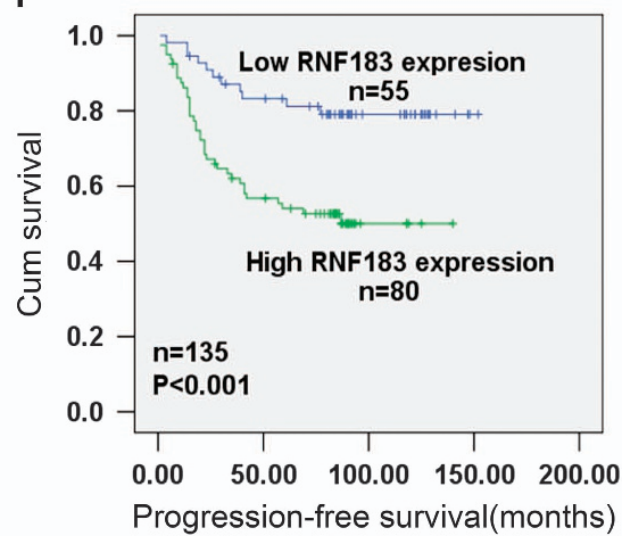

Figure 1 The expression of RNF183 is elevated in human colorectal cancer tissues. (a) Reanalyzing the expression of RNF family members between normal and tumor tissues in CRC from GEO data set GSE8671. (b) The relative mRNA expression of RNF183 was evaluated by RT-PCR in 15 paired human normal colorectal tissues and CRC tissues. (c) IHC staining of RNF183 expression in CRC tissues and adjacent normal mucosal tissues. Scale bar: $100 \mu \mathrm{m}$ ( $\times 10)$ or $50 \mu \mathrm{m} \times(40)$. (d) IHC analyses of RNF183 in paired CRC cancerous and noncancerous tissues from 135 patients. (e and f) Kaplan-Meier survival analysis of the association between RNF183 expression and overall survival (e) or progression-free survival (f) in 135 patients. $P$-value was determined by long rank test. ${ }^{* \star *} P<0.001$

\section{Results}

RNF183 is upregulated in CRC. To identify RNF members that are aberrantly expressed in CRC, we compared gene expression of 202 RNF genes between cancerous and normal adjacent tissues using GEO data set GSE8671. The analysis showed that 70 RNF family members are elevated in CRC tissues (Figure 1a). The top two genes among these upregulated RNF members, RNF206/TRAIP and RNF90/ TRIM7 have been reported to drive tumor formation. ${ }^{15,16}$ RNF183 ranks the third in all elevated genes with unknown function in CRC, and was chosen for further analysis. Quantitative RT-PCR experiment in 15 paired CRC and corresponding normal tissues confirmed elevated RNF183 mRNA level in cancerous tissues (Figure 1b). Furthermore, we detected the level of RNF183 protein in 135 paired CRC 
tissues using immunohistochemistry $(\mathrm{IHC})$, and the staining was scored for each sample (four intensity levels: 0-3; Supplementary Figure 1). The expression of RNF183 is significantly higher in tumor tissues than in adjacent nontumor tissues (Figures 1c and d). High and low RNF183 staining was observed in 80 (59.3\%) and 55 (40.7\%) CRC tissue samples, respectively (Table 1). RNF183 high expression was significantly associated with tumor size $(P=0.012)$, tumor invasive depth $(P=0.004)$, TNM stage $(P=0.01)$, and distant metastasis $(P=0.09)$ (Table 1$)$. Kaplan-Meier survival analysis showed that patients with high RNF183 expression had shorter overall survival (OS, Figure 1e) and progress-free survival (PFS, Figure 1f). Multivariate Cox proportional hazards regression analysis showed that RNF183 level is an independent prognostic factor for PFS $(P<0.05$, hazard's ratio $(\mathrm{HR})=0.384,95 \% \mathrm{Cl}=0.172-0.861)$ and $\mathrm{OS}(P<0.05$, $\mathrm{HR}=0.364,95 \% \mathrm{Cl}=0.159-0.831)$ (Table 2).

RNF183 accelerates the growth of CRC cells. As unlimited growth, invasiveness, and metastasis are hallmarks of malignancy, ${ }^{17}$ we tried to explore the role of RNF183 in progression of CRC. The endogenous RNF183 expression is higher in CRC cells than in NCM460, a normal colorectal epithelial cell (Figure 2a). We silenced RNF183 expression in DLD-1 and HCT116 cells, and the knockdown efficiency is shown in Figure 2b. RNF183 knockdown suppressed the growth of DLD-1 and HCT116 cells (Figure 2c), whereas RNF183 overexpression accelerates the proliferation of both cell lines in MTT assays (Figure 2d). Furthermore, colony formation assay shows that RNF183 silencing significantly reduced size and number of colonies (Figure $2 e$ ), and its enforced expression shows opposite phenomenon (Figure 2f). These results indicated that RNF183 promotes cell growth in vitro.

We next investigated whether RNF183 affects the proliferation of CRC cells in vivo. Control and RNF183 stable overexpression DLD-1 cells were subcutaneously injected into BALB/c nude mice to generate xenograft tumors. The results showed that RNF183 enforced expression significantly promote tumor growth (Figure 2g). Tumors with RNF183 overexpression have larger size and higher weight than control tumors $(P<0.01)$ (Figure $2 \mathrm{~h})$. RNF183 overexpression was confirmed by $\mathrm{IHC}$ staining (Figure $2 \mathrm{i}$ ), and tumors with RNF183 overexpression have higher percentage of Ki-67-positive cells than control tumors (Figures 2i and j).

RNF183 promotes migration, invasion, and metastasis of CRC cells. Subsequently, we explored the function of RNF183 on the motility of CRC cells. In transwell assays, RNF183 knockdown significantly impaired the migration and invasion ability of HCT116 (Figure 3a) and DLD-1 (Figure 3b) cells, whereas RNF183 overexpression largely facilitated the motility of both cell lines (Figures $3 c$ and $d$ ). Given that epithelial-mesenchymal transition (EMT) is a critical event involved in the initiation of tumor invasion, the impact of RNF183 on the expression of several EMT markers was analyzed in HCT116 cells. RNF183 overexpression significantly induced the transcription of Snail and ZEB1 and repressed the mRNA abundance of ZO-1 and E-cadherin (Figure 3e, upper). RNF183 knockdown, however, repressed
Table 1 Clinicopathological characters and their correlation with RNF183 expression

\begin{tabular}{|c|c|c|c|c|}
\hline Variables & $N(\%)$ & $\begin{array}{c}\text { RNF183 low } \\
(\%)\end{array}$ & $\begin{array}{l}\text { RNF183 } \\
\text { high (\%) }\end{array}$ & $P$-value \\
\hline Total case & 135 & $55(40.7 \%)$ & $80(59.3 \%)$ & \\
\hline \multicolumn{5}{|l|}{ Age (years) } \\
\hline$<65$ & $91(67.4 \%)$ & $36(26.7 \%)$ & $55(40.7 \%)$ & \\
\hline$\geqslant 65$ & $44(32.6 \%)$ & $19(14.1 \%)$ & $25(18.5 \%)$ & 0.726 \\
\hline \multicolumn{5}{|l|}{ Gender } \\
\hline Male & $76(56.3 \%)$ & $29(21.5 \%)$ & $47(34.8 \%)$ & \\
\hline Female & $59(43.7 \%)$ & $26(19.3 \%)$ & $33(24.4 \%)$ & 0.492 \\
\hline \multicolumn{5}{|l|}{ Tumor location } \\
\hline Colon & $64(47.4 \%)$ & $23(17 \%)$ & $41(30.4 \%)$ & \\
\hline Rectum & $71(52.6 \%)$ & $32(23.7 \%)$ & $39(28.9 \%)$ & 0.284 \\
\hline \multicolumn{5}{|c|}{ Tumor size $(\mathrm{cm})$} \\
\hline$<5$ & $54(40 \%)$ & $29(21.5 \%)$ & $25(18.5 \%)$ & \\
\hline$\geqslant 5$ & $81(60 \%)$ & $26(19.3 \%)$ & $55(40.7 \%)$ & $0.012^{a}$ \\
\hline \multicolumn{5}{|c|}{ Tumor invasive depth } \\
\hline $\mathrm{T} 1-\mathrm{T} 2$ & $24(17.8 \%)$ & $16(11.8 \%)$ & $8(6 \%)$ & \\
\hline T3-T4 & $111(82.2 \%)$ & $39(28.9 \%)$ & $72(53.3 \%)$ & $0.004^{\mathrm{a}}$ \\
\hline \multicolumn{5}{|c|}{ AJCC/TNM stage } \\
\hline I-II & $63(46.7 \%)$ & $33(24.4 \%)$ & $30(22.3 \%)$ & \\
\hline III-IV & $72(53.3 \%)$ & $22(16.3 \%)$ & $50(37 \%)$ & $0.01^{a}$ \\
\hline \multicolumn{5}{|c|}{ Lymph node status } \\
\hline$<1$ & $68(50.4 \%)$ & $35(26 \%)$ & $33(24.4 \%)$ & \\
\hline$\geqslant 1$ & $67(49.6 \%)$ & $20(14.8 \%)$ & 47 (34.8\%) & 0.35 \\
\hline \multicolumn{5}{|c|}{ Distant metastasis } \\
\hline $\begin{array}{l}\text { No } \\
\text { metastasis }\end{array}$ & $105(77.8 \%)$ & $49(36.3 \%)$ & $56(41.5 \%)$ & \\
\hline Metastasis & $30(22.2 \%)$ & $6(4.4 \%)$ & $24(17.8 \%)$ & $0.009^{a}$ \\
\hline \multicolumn{5}{|c|}{ Preoperative CEA (ng/ml) } \\
\hline$<5$ & $64(50.4 \%)$ & $29(22.8 \%)$ & $35(27.6 \%)$ & \\
\hline$\geqslant 5$ & $63(49.6 \%)$ & $24(18.9 \%)$ & $39(30.7 \%)$ & 0.414 \\
\hline
\end{tabular}

Abbreviations: CA199, carbohydrate antigen 19-9; CEA, carcino embryonic antigen

The numbers in parentheses indicate the percentages of tumors with a specific clinical or pathologic feature for a given RNF183 subtype

astatistically significant, $P<0.05$

Snail and ZEB1 expression but induced ZO-1 and E-cadherin transcription (Figure $3 e$, bottom). The regulation of these EMT markers was further confirmed by western blots (Figure 3f).

The above in vitro results encouraged us to evaluate the role of RNF183 on tumor metastasis in vivo. We developed lung and hepatic metastasis models by injecting control or RNF183 stable overexpression DLD-1 cells into the lateral tail veins or spleens of nude mice for 8-10 weeks. Compared with control groups, RNF183 enforced expression increased the number of metastatic pulmonary nodules (Figure 4a) and liver nodules (Figure 4b). In knockdown experiments, RNF183 silencing reduced lung (Figure 4c) and hepatic (Figure $4 d$ ) metastasis of HCT116 cells. These data suggested that RNF183 promotes migration, invasion and metastasis of $\mathrm{CRC}$ cells in vitro and in vivo.

RNF183 regulates IL-8 transcription through NF- $\kappa$ B signaling. The function of RNF183 in growth and metastasis of $\mathrm{CRC}$ cells predicts its role in regulating oncogenic 
pathways. It had been reported that RNF183 activates NF- $\kappa$ B in $I B D,{ }^{12}$ so we performed $\mathrm{APCR}$ to evaluate the influence of RNF183 on several NF- $k$ B downstream targets. RNF183 knockdown repressed the transcription of $I L-8, I L-6, C O X 2$, iNOS as well as $I L-1 \beta$, and the reduction of $I L-8$ is the most significant among these genes (Figure 5a). ELISA assays confirmed that RNF183 knockdown markedly decreased the level of secreted IL-8 in HT116 and DLD-1 cells (Figure 5b). On the contrary, RNF183 enforced expression significantly induced IL-8 level at both mRNA and protein level in DLD-1 cells (Figure $5 \mathrm{c}$ ). Elevated $I L-8$ transcription was also observed in DLD-1 xenograft tumors with RNF183 overexpression (Figures $2 \mathrm{~g}$ and $5 \mathrm{~d}$ ).

In order to elucidate whether NF- $\kappa$ B is required for RNF183 to induce $I L-8$ transcription, we performed luciferase assays using wild-type IL-8 promoter (IL-8-Luc) or IL-8 promoter with NF- $\kappa$ B binding site deletion (IL-8- $\triangle N F-\kappa$ B-Luc). As shown in Figure 5e, RNF183 activates IL-8-Luc but not IL-8- $\triangle N F-\kappa B-$ Luc, suggesting that NF- $\kappa \mathrm{B}$ is indispensable for RNF183 to regulate $I L-8$ transcription. Furthermore, we assessed the effect of RNF183 on the expression of several key proteins in $\mathrm{NF}-\kappa \mathrm{B}$ pathway. Among all tested proteins, P65 was significantly suppressed or evaluated according to RNF183 knockdown or overexpression (Figure 5f), respectively. As $\mathrm{P} 65$ is a core transcriptional factor drives $I L-8$ expression, we detected the enrichment of this protein on $I L-8$ promoter. Chromatin immunoprecipitation (ChIP) assays were performed and increased binding of P65 on IL-8 promoter was found upon RNF183 enforced expression (Figure $5 \mathrm{~g}$ ). Finally, $\mathrm{IHC}$ staining showed a positive correlation of RNF183 and P65 protein level in $40 \mathrm{CRC}$ tissue sections (Figure 5h). Taken together, these data suggest that RNF183 induced P65 expression and subsequent NF- $\kappa \mathrm{B}$ activation is responsible to stimulate $I L-8$ transcription in CRC cell, xenograft and pathological tissues.
The oncogenic function of RNF183 is dependent on NF- $\boldsymbol{\kappa}$ B-IL-8 axis. Encouraged by the above results, we decided to challenge whether targeting NF- $\kappa \mathrm{B}-\mathrm{IL}-8$ axis could attenuate the oncogenic function of RNF183. We treated DLD-1 cells with NF- $\kappa$ B inhibitor BAY11-7085, and found that this compound could inhibit RNF183 overexpression induced cell migration (Figure 6a) and invasion (Figure $6 \mathrm{~b}$ ). In another assay, transfection of IL-8 targeted siRNA inhibits RNF183 induced migration of DLD-1 cells (Figure 6c), and reduced IL-8 secretion is confirmed by ELISA assay (Supplementary Figure 2). As expected, this phenomenon was rescued by adding recombinant IL-8 protein to the culture medium of knockdown cells (Figure $6 \mathrm{c}$ ), suggesting an oncogenic role of IL-8 as previously reported. ${ }^{18,19}$ Next, we generated a truncated form of RNF183 (RNF183-mut) without E3 ubiquitin ligase activity by deleting its RING domain (amino acids 1-59), and compared its function with wild-type RNF183. This truncation largely abolished the function of RNF183 in activating IL-8 promoter (Figure $6 \mathrm{~d}$ ) and promoting the growth (Figure 6e), colony formation (Figure 6f), and migration (Figure $6 \mathrm{~g}$ ) of HCT116 cells. These results thus suggest that the oncogenic function of RNF183 is largely dependent on its E3 ubiquitin ligase activity to regulate NF- $\kappa$ B-IL-8 axis.

\section{Discussion}

Overwhelming evidences have shown that inflammation has crucial roles in tumor development, including initiation promotion, malignant conversion, invasion, and metastasis. ${ }^{20}$ Studies even show that inflammatory responses can eliminate the effects of cancer therapy. ${ }^{21}$ A classic inflammation-driven cancer is a subtype of CRC named colitis-associated colon cancer (CAC), which is usually developed from IBD. ${ }^{14,22}$ Other subtypes of colorectal tumor also demonstrate vigorous

Table 2 Multivariate analysis for PFS and OS in CRC patients

\begin{tabular}{|c|c|c|c|c|}
\hline Variables & PFS HR (95\% Cl) & $P$-value & OS HR $(95 \% \mathrm{Cl})$ & $P$-value \\
\hline Age (year, $<65$ versus $\geqslant 65$ ) & $0.692(0.355-1.349)$ & 0.28 & $0.6(0.34-1.316)$ & 0.244 \\
\hline Gender (male versus female) & $0.94(0.482-1.833)$ & 0.856 & $0.96(0.484-1.907)$ & 0.908 \\
\hline Tumor location (colon versus rectum) & $0.724(0.38-1.38)$ & 0.327 & $0.69(0.362-1.342)$ & 0.28 \\
\hline Tumor size $(\mathrm{cm},<5$ versus $\geqslant 5)$ & $0.811(0.371-1.771)$ & 0.599 & $0.86(0.391-1.89)$ & 0.707 \\
\hline Tumor invasive depth (T1-2 versus T3-4) & $2.733(0.672-11.111)$ & 0.16 & $2.805(0.692-11.372)$ & 0.14 \\
\hline Lymph node status $(<1$ versus $\geqslant 1)$ & $1.062(0.427-2.641)$ & 0.897 & $0.957(0.382-2.401)$ & 0.926 \\
\hline Distant metastasis (no versus yes) & $0.192(0.086-0.432)$ & $<0.001^{a}$ & $0.152(0.067-0.347)$ & $<0.001^{\mathrm{a}}$ \\
\hline Stage & $0.145(0.036-0.585)$ & $0.007^{\mathrm{a}}$ & $0.166(0.041-0.673)$ & $0.012^{\mathrm{a}}$ \\
\hline Preoperative CEA (ng/ml $<5$ versus $\geqslant 5$ ) & $0.768(0.407-1.447)$ & 0.414 & $0.69(0.364-1.308)$ & 0.256 \\
\hline RNF183 (high versus low) & $0.384(0.172-0.861)$ & $0.02^{\mathrm{a}}$ & $0.364(0.159-0.831)$ & $0.016^{\mathrm{a}}$ \\
\hline
\end{tabular}

Abbreviations: CEA, carcino embryonic antigen; $\mathrm{Cl}$, confidence interval; HR, hazard ratio; OS, overall survival; PFS, progression-free survival

a Statistically significant $P<0.05$

Figure 2 RNF183 promotes proliferation of CRC cells in vitro and in vivo. (a) Western blots detect endogenous RNF183 protein expression in human normal colorectal epithelial cell line and CRC cells. (b) RNF183 knockdown efficiency in two CRC cell lines was levels were examined by western blots. (c and d) Effects of RNF183 silencing (c) or overexpression (d) on proliferation of HCT116 and DLD-1 cells was monitored by MTT assays. Mean \pm S.D. ( $n=6)$. (e and f) Effects of RNF183 silencing (e) or enforced expression (f) on the colony formation of HCT116 and DLD-1 cells. (g-j) RNF183 overexpression accelerates tumor growth in vivo. (g) Tumor pictures from mice inoculated with stable RNF183 overexpression cell line DLD-1 or control. (h) Growth curve of tumor volume measured on indicated days (left) and tumor weight at the end of experiment (right). Mean \pm S.D. $(n=6)$. (i) Photographs exhibited the H\&E staining (left), IHC staining for RNF183 (middle) and ki-67 (right) in tumors. Scale bar: $100 \mu$ m. (j) Number of Ki-67 positive cells in control and RNF183 stable expression tumors. ${ }^{*} P<0.05,{ }^{\star \star} P<0.01,{ }^{\star * \star} P<0.001$ 
inflammation and aberrant high level of inflammatory cytokines. $^{23,24}$ Moreover, a large proportion of CRC tumors and $\mathrm{CRC}$ cell lines exhibits increased activity of NF- $\kappa \mathrm{B}$ signal pathway. ${ }^{25}$ However, the underlying molecular mechanism that drive the hyperactivation of inflammatory responses in $\mathrm{CRC}$ remains largely unknown. a
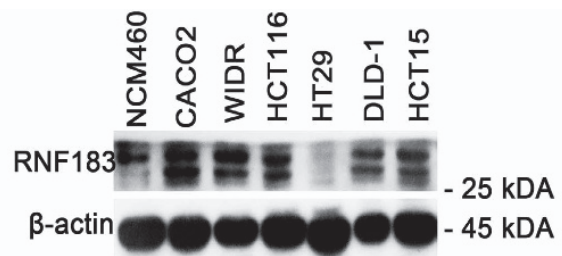

C
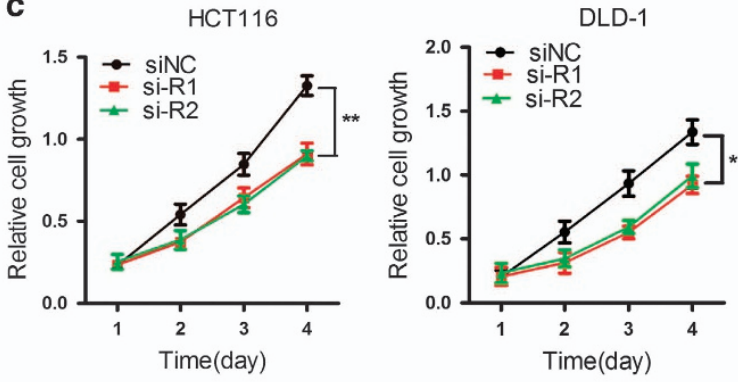

e
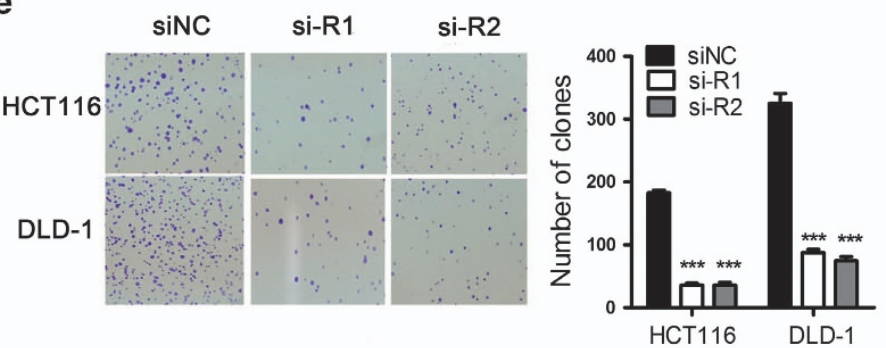

h
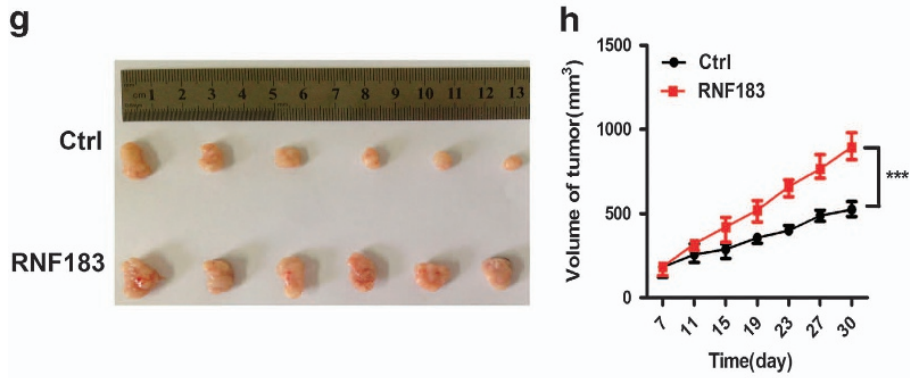

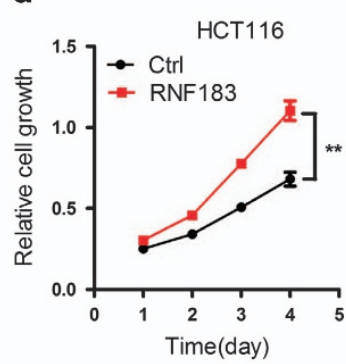

f

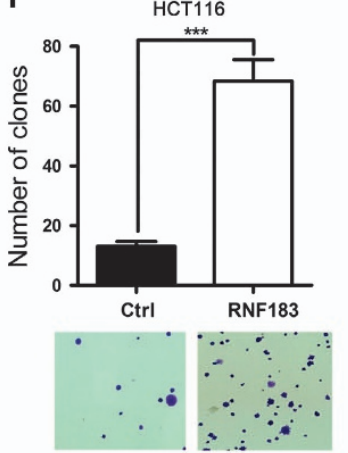

\section{d}

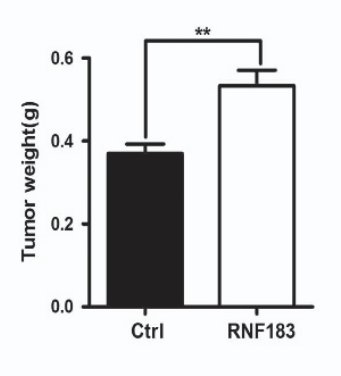

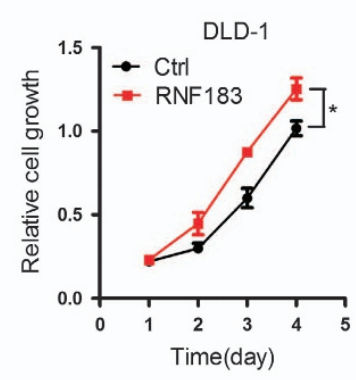

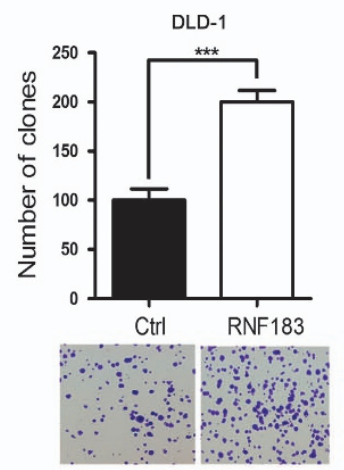

j

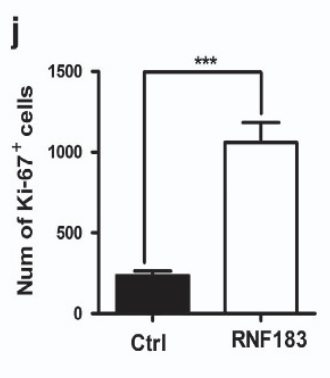

- 25 kDA

- 45 kDA

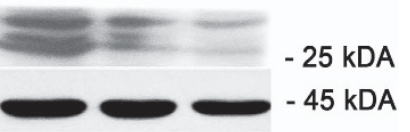

i

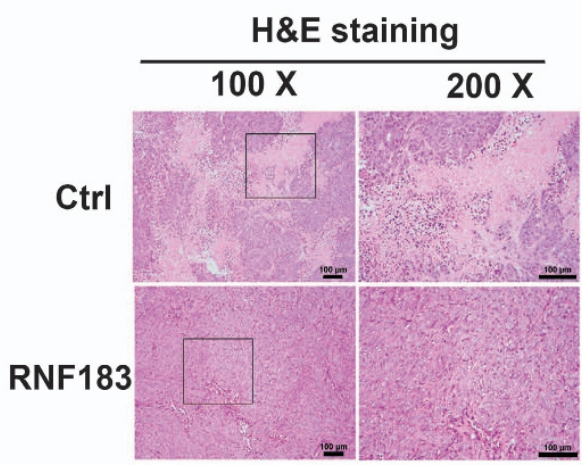

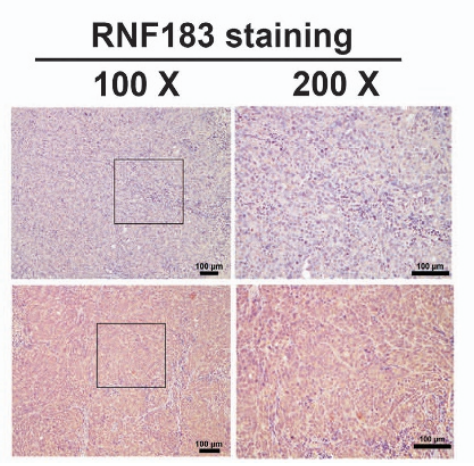


a
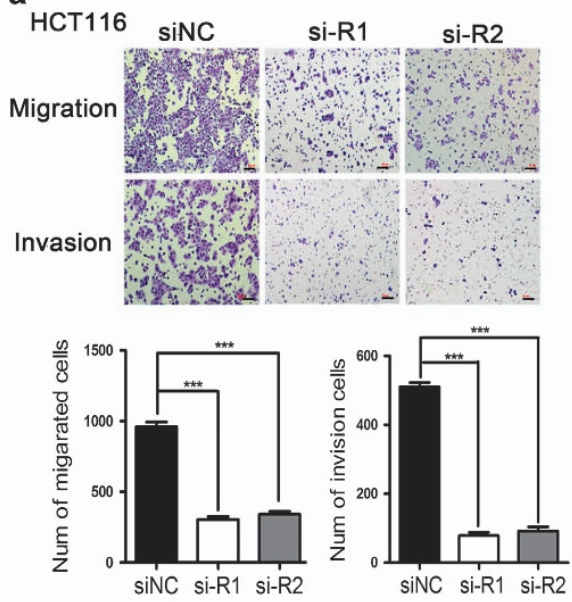

c
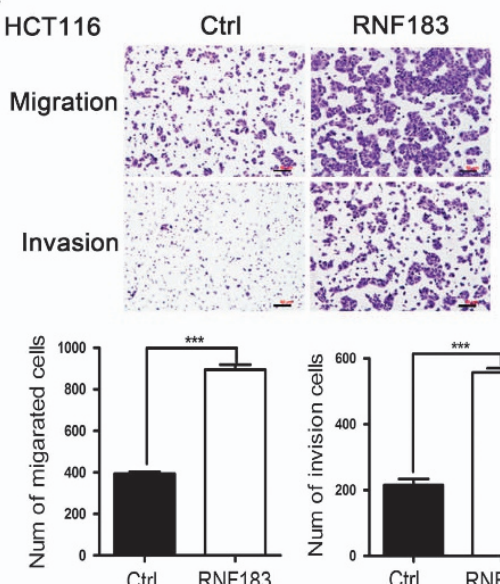

Ctrl RNF183 b
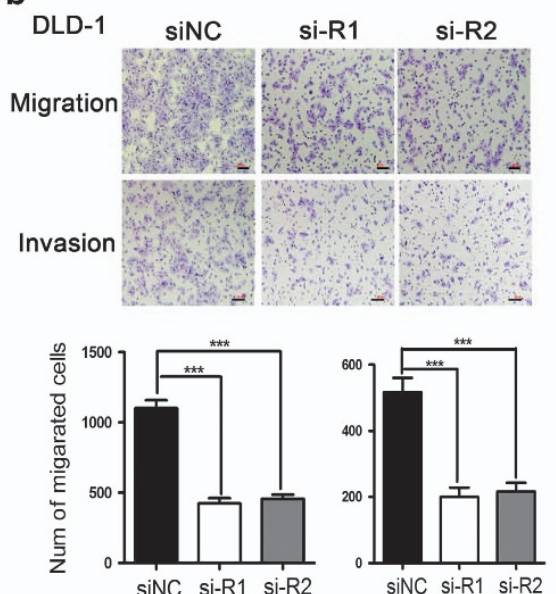

d
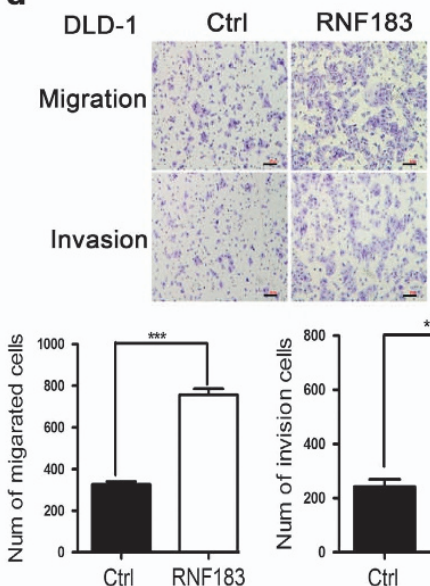

e

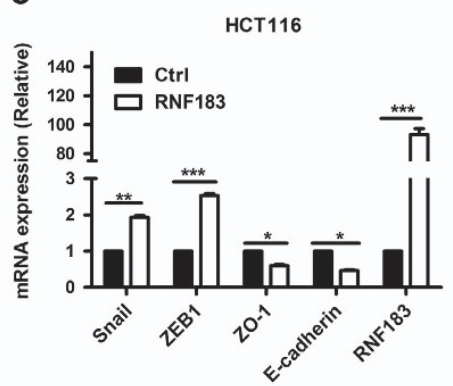

HCT116

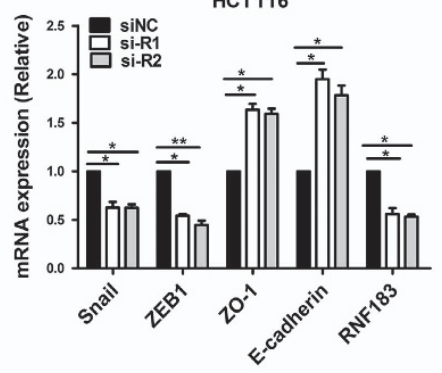

f

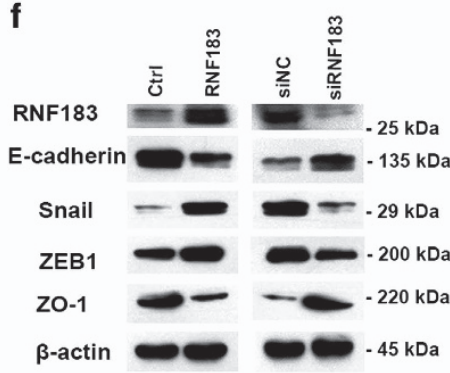

Figure 3 RNF183 prmotes migration, invasion and epithelial-mesenchymal transition (EMT) of CRC cells. (a and b) Effects of RNF183 silencing on migration and invasion of HCT116 (a) and DLD-1 (b) cells evaluated by transwell assays. Mean \pm S.D. $(n=3)$. (c and d) Effects of RNF183 overexpression on migration and invasion of HCT116 (c) and DLD-1 (d) cells detected by transwell assays. Mean \pm S.D. $(n=3)$. (e) Effects of RNF183 overexpression (upper) and silencing (bottom) on the mRNA abundance of selected EMT markers in HCT116 cells. (f) Effects of RNF183 overexpression (left) and silencing (right) on the protein expression of selected EMT markers in HCT116 cells. Scale bar: $100 \mu \mathrm{m} .{ }^{\star} P<0.05,{ }^{* \star} P<0.01,{ }^{* \star *} P<0.001$

Previous study indicated that RNF183 is upregulated in intestinal epithelial cells of IBD patients and TNBS-induced colitis mice. ${ }^{12}$ In our study, we provided evidences to show that RNF183 is aberrantly overexpressed in CRC cell lines and tissues. Patients with high expression of RNF183 have shorter OS and PFS, indicating that RNF183 could serve as an independent prognostic factor in CRC. Enforced expression of RNF183 promotes proliferation, invasion, and metastasis of tumor cells, whereas opposite phenomenon were observed in RNF183 knockdown experiments, indicating that RNF183 functions as an oncogene in the pathological process of CRC. Elevated expression of RNF183 in both IBD and CRC suggested a possibility that RNF183 may contribute to the transformation from inflammation to malignancy. In IBD, RNF183 activates NF- $\kappa$ B pathway through ubiquitination and degradation of $\mathrm{I}_{\kappa} \mathrm{B} a{ }^{12}$ In CRC cells, we found that RNF183 induced IL-8 expression in an E3 ubiquitin ligase-dependent manner. IL-8 is transcriptional controlled by transcription factors NF- $\kappa$ B and AP-1, ${ }^{18}$ and we confirmed that RNF183 increased the abundance of NF- $\kappa \mathrm{B}$ transcription factor P65 and its enrichment to $I L-8$ promoter in CRC cells. In accordance with previous reports that $\mathrm{NF}-\kappa \mathrm{B}$ signal pathway and its downstream chemokine IL-8 contributes to inflammation and to every step of CRC progression proliferation, migration and invasion, ${ }^{26-28}$ we found that NF- $\kappa$ B inhibition and IL-8 depletion eliminates the tumor-promoting activity of RNF183.

Taken together, our data indicate that RNF183 contributes to the transformation from inflammation to malignancy in $\mathrm{CRC}$, and predicts the application of NF- $\kappa \mathrm{B}$ inhibitors or IL-8 antagonists for precision therapy of CRC with elevated RNF183 expression.

\section{Materials and Methods}

Bioinformatics analyses. The expression of RNF family genes were downloaded from GEO database (https:/www.ncbi.nlm.nih.gov/geo/) under the accession number GSE8671. The significance of gene expression between normal and tumor tissues were calculated using Excel 2007. The heatmap was drawn using 
a H\&E staining
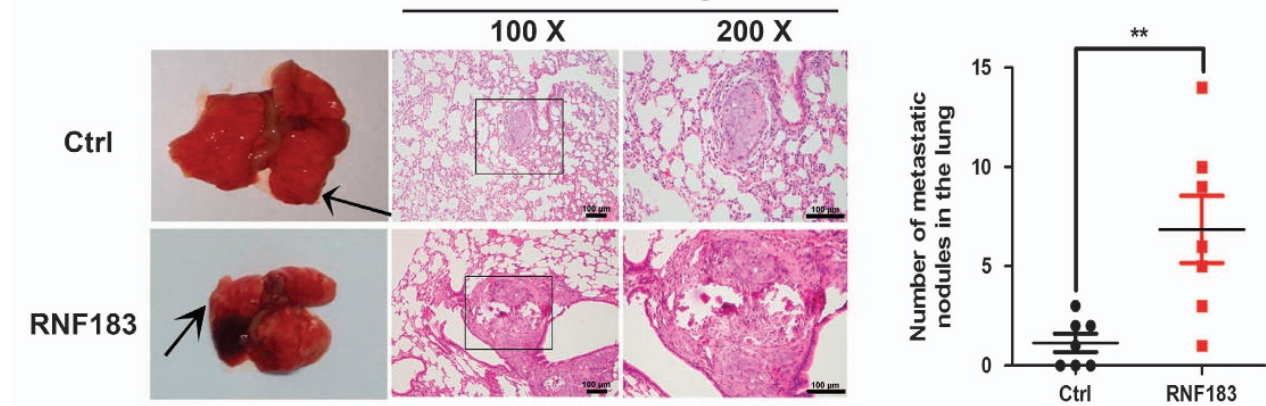

b

H\&E staining
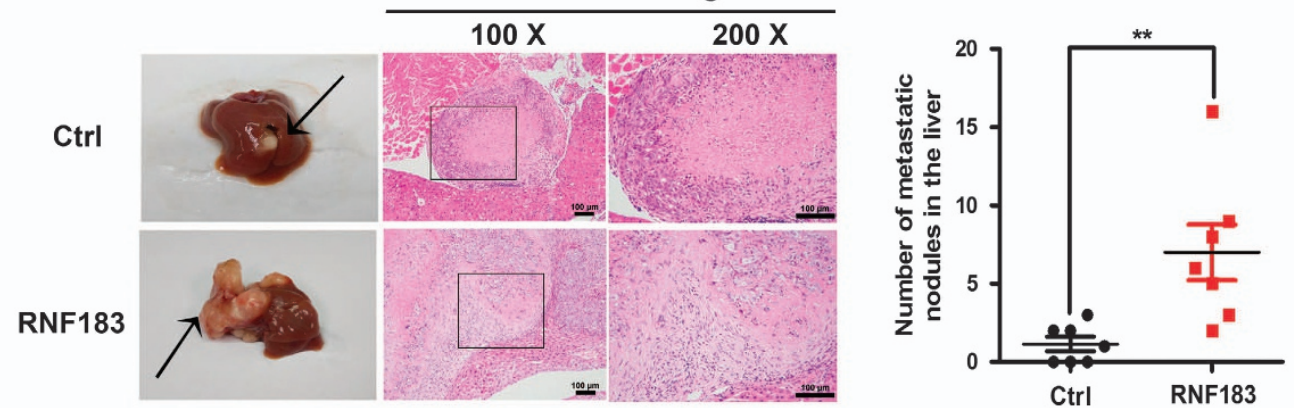

C

H\&E staining
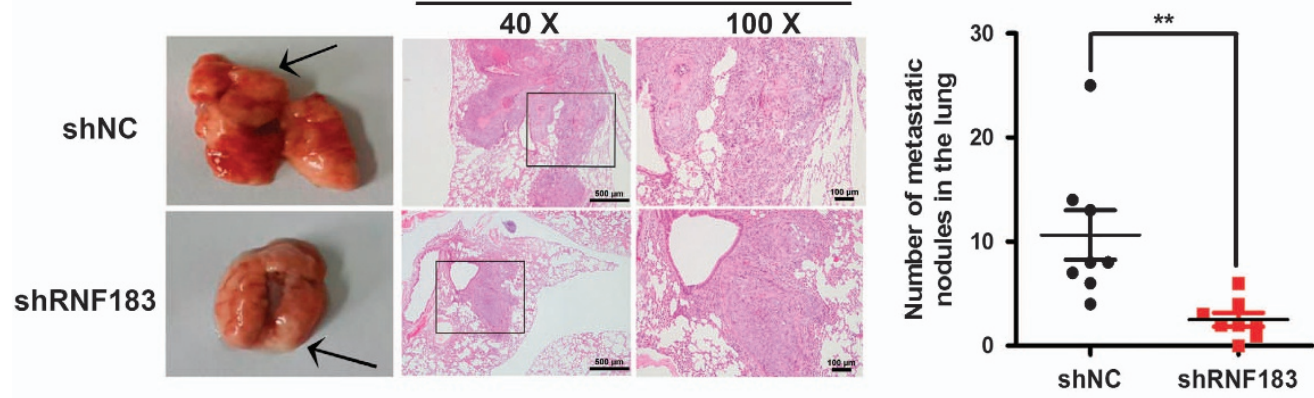

d
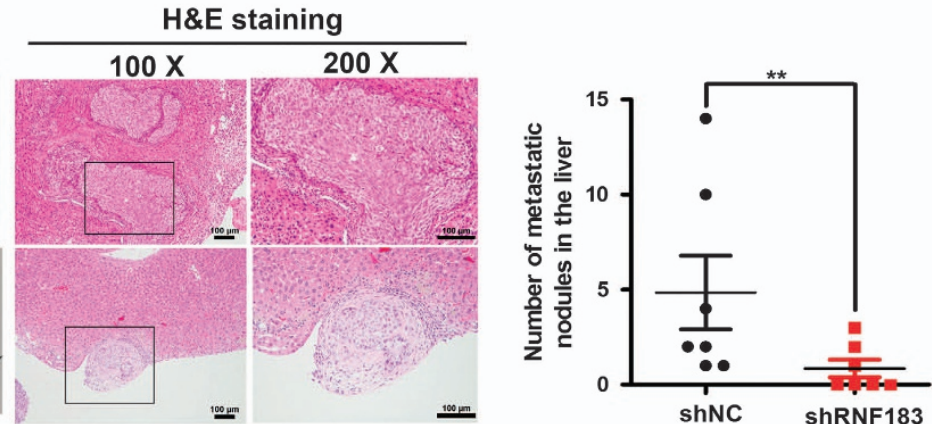

Figure 4 RNF183 promotes metastasis of CRC cells in vivo. (a) Lungs were removed from mice injected with control or DLD-1 stable cell line with RNF183 overexpression. Pulmonary histopathology was examined by H\&E staining. Mean \pm S.D. $(n=7)$. (b) Livers were removed from mice injected with control or DLD-1 stable cell line with RNF183 overexpression. Hepatic histopathology was examined by H\&E staining. Mean \pm S.D. $(n=7)$. (c) Lungs were dissected from mice injected with control or RNF183 stable silenced HCT116 cells. Pulmonary histopathology was examined by H\&E staining mean \pm S.D. $(n=8)$. (d) Livers were dissected from mice injected with control or RNF183 stable silenced HCT116 cells. Hepatic histopathology was examined by H\&E staining. Mean \pm S.D. $(n=7)$. Scale bar: $100 \mu \mathrm{m}(\times 100$ and $\times 200)$ or $500 \mu \mathrm{m}(\times 40)$. ${ }^{\star *} P<0.01$

Genesis software (http://genome.tugraz.at/genesisclient/genesisclient_description. shtml). For genes with multiple probe-sets, the one with lowest $P$-value between normal and tumor tissues were chosen for further analyses.

Plasmids, antibodies, and reagents. The plasmid of pcDNA4-myc/hisRNF183 was constructed by the company of genewiz (Suzhou, China). A truncated form of RNF183 without amino acids 1-59 was generated by PCR and subsequent molecular cloning into pcDNA4-myc/his vector. IL-8 and IL-8- $\triangle$ NF- $\kappa$ B reported plasmids were kindly provided by Professor Hongbin Shu (Wuhan University, China). The antibodies used were listed as follows: human anti-RNF183 antibody (1:1000 for western blot, 1:200 for IHC, ab197321, Abcam, Cambridge, UK), antiTAB1 antibody (1;1000, \#3226 CST), anti-TRAF2 antibody (1:200, sc-136999, 
a

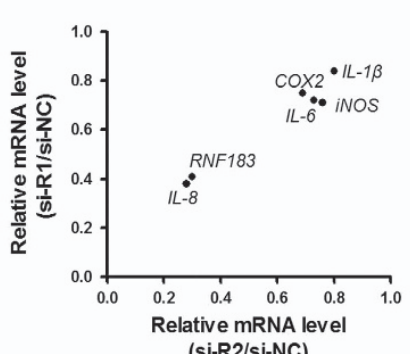

(si-R2/si-NC)

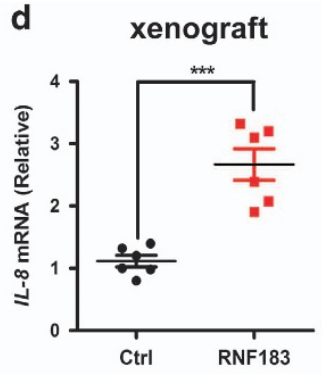

e

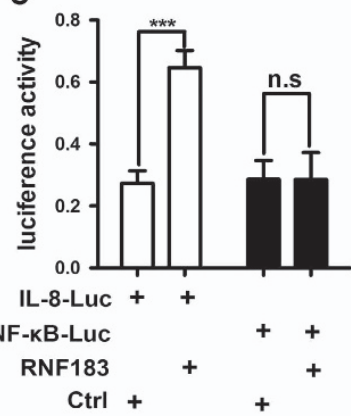

b
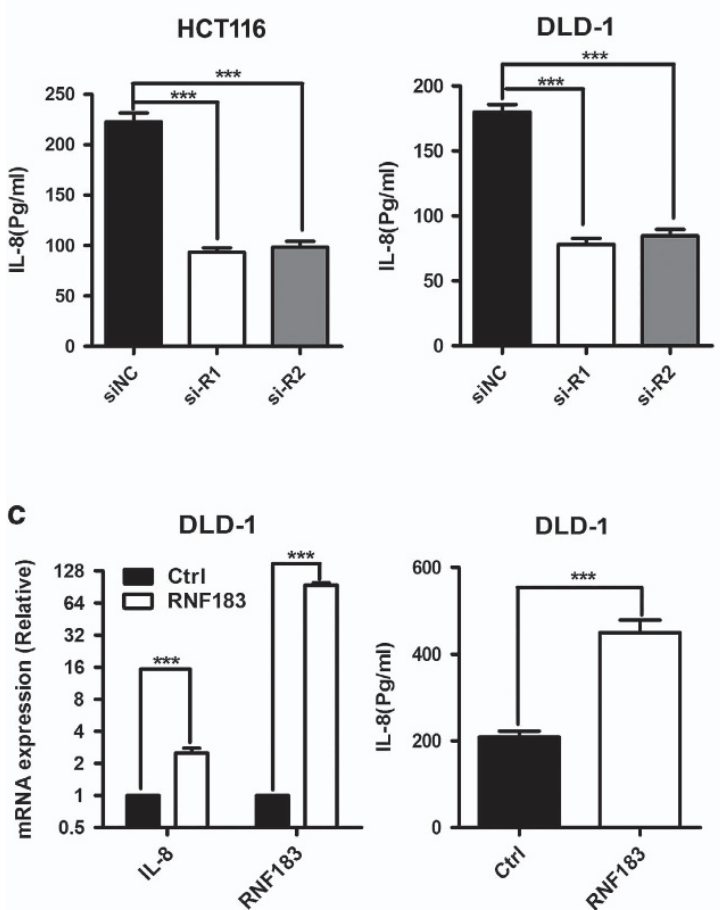
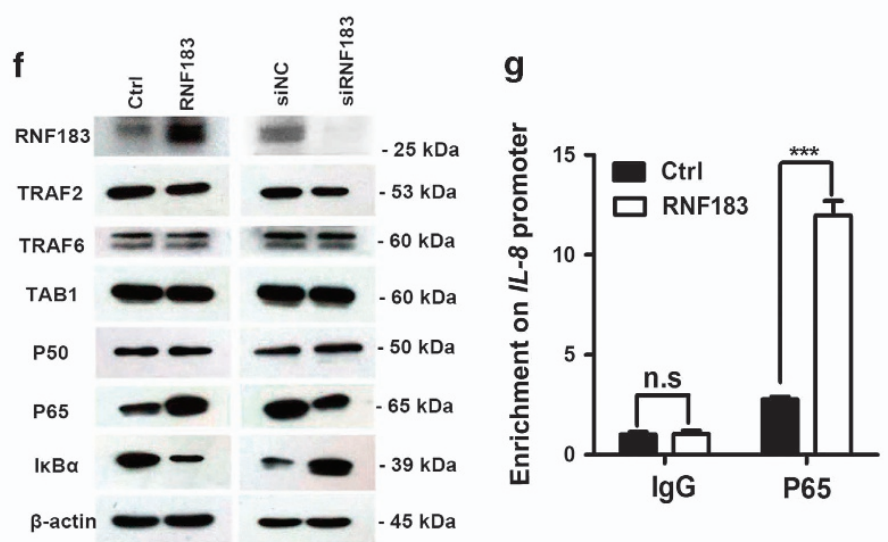

h
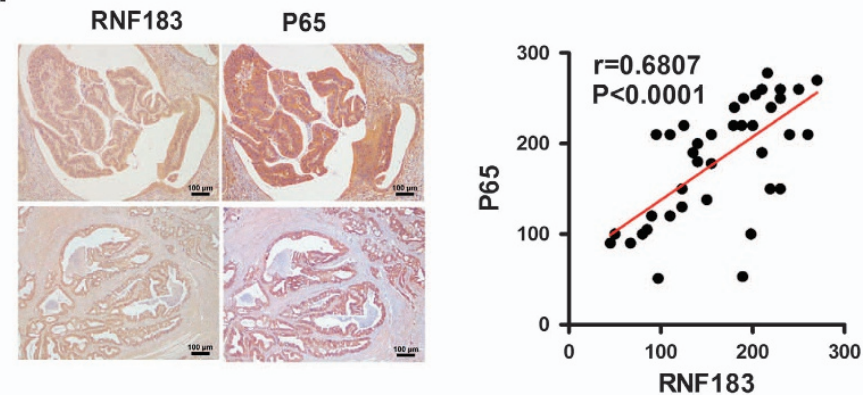

Figure 5 RNF183 promotes IL-8 transcription through NF- $k$ B. (a) The effects of RNF183 knockdown on the mRNA abundance of several NF- $k B$ downstream genes in HCT116 cells. (b) Knockdown RNF183 expression significantly reduced IL-8 secretion in HCT116 (left) and DLD-1 (right) cells. (c) Enforced RNF183 expression augments IL-8 transcription (left) and IL-8 secretion (right) in DLD-1 cells. (d) Stable RNF183 overexpression increased IL-8transcription in xenograft tumors as shown in Figure 2g. (e) Effects of RNF183 on the activity of luciferase reporter with wild-type or NF- $\kappa B$ binding site deleted ( $\triangle N F-\kappa B) ~ I L-8$ promoter in HCT116 cells. (f) Expression of several proteins in NF- $\kappa B$ pathway was examined by western blots with enforced RNF183 expression in HCT116 cells or with RNF183 knockdown in DLD-1 cells. (g) Chromatin immunoprecipitation (ChIP) assays were carried out to determine the binding of P65 on IL-8 promoter with or without RNF183 enforced expression. (h) The expression of P65 and RNF183 were evaluated in forty CRC tissues. The correlation of these two proteins and the significance were also calculated. Mean \pm S.D. $(n=3)$. Scale bar: $100 \mu \mathrm{m} .{ }^{* \star *} P<0.001$

Santa Cruz Biotech, Santa Cruz, CA, USA), anti-TRAF6 (1:200, sc-7221, Santa Cruz), anti-NF- $\kappa B$ p65 antibody (1:1000 for western blot, 1:200 for IHC, 1:100 for CHIP, \#8242, CST), anti-NF- $\kappa B$ P50 antibody (1:1000, \#12540, CST, Boston, MA, USA), anti-ki-67 antibody(1:1000, \#12202, CST), anti- $\beta$-actin (1:200, sc-47778, Santa Cruz), anti-mouse IgG HRP conjugate (1:8000, W402B, Promega, Madison, WI, USA), anti-Rabbit IgG HRP conjugate (1:2000, \#7074P2, CST), normal rabbit IgG ( $1 \mu \mathrm{g}$ for ChIP, sc-3888, Santa Cruz). Recombinant human IL-8 was purchased from Novus (Littleton, CO, USA) (NBP2-34905). The siRNAs (ribobio Company, Beijing, China) sequences were listed in supplementary table 1.

Cell lines and cell culture. All CRC cell lines were purchased from the American Type Culture Collection (ATCC) and cultured in a humidified atmosphere of $5 \% \mathrm{CO}_{2}$ at $37^{\circ} \mathrm{C}$. $\mathrm{HCT} 116$ and $\mathrm{HT} 29$ were cultured in McCoy's $5 \mathrm{~A}$ Medium (MC5A, Gibco, Carlsbad, CA, USA); NCM460, CACO2, and WIDR were cultured in
Dulbecco's Modified Eagle Medium; DLD-1 and HCT15 were cultured in RPMI 1640 medium. A total of $10 \%$ fetal bovine serum (FBS) was supplemented in the culture medium. Thawed cells from liquid nitrogen were used for within the first three passages.

Patient samples. Fifteen $\mathrm{CRC}$ specimens and their matched adjacent normal tissue (for RNF183 mRNA level analysis by RT-PCR) were obtained from Sun Yat-sen university cancer center (Guangzhou, China) with the agreement of the patients. A total of $135 \mathrm{CRC}$ patients who underwent resection of primary CRC between January 1999 and December 2005 at the Sun Yat-sen University Cancer Center were chosen for IHC assays. The detailed selection criterion for CRC patients was described previously. ${ }^{29}$ 
a

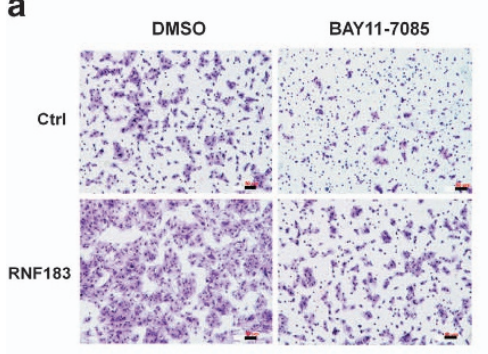

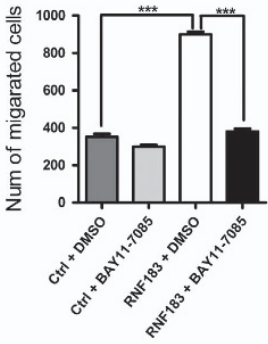

C

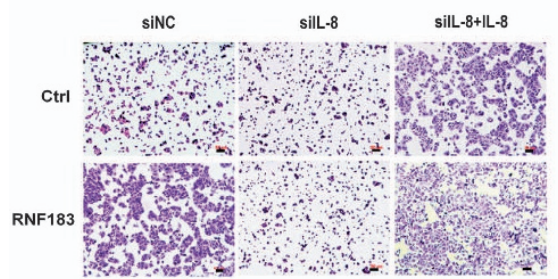

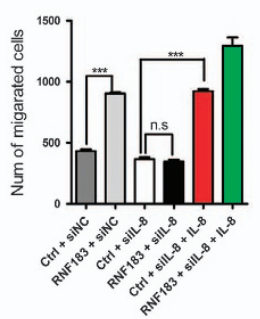

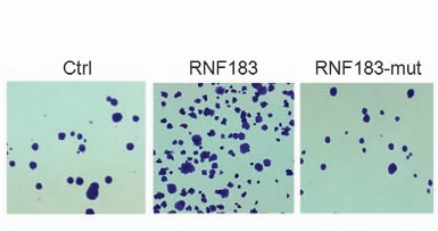

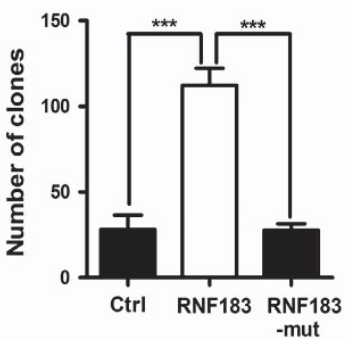

b
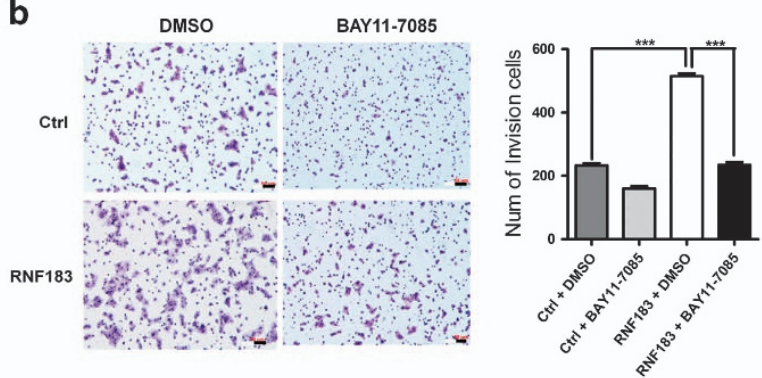

d
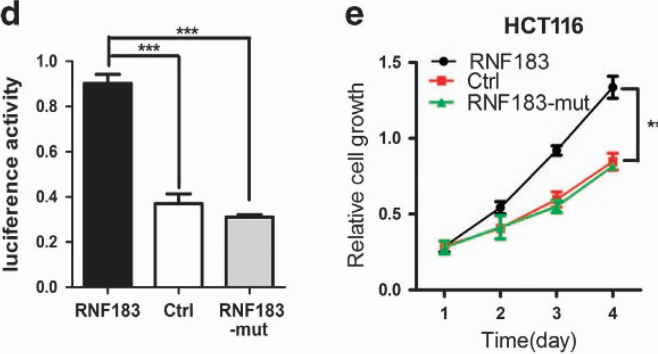

g

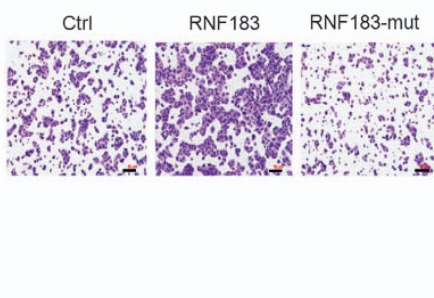

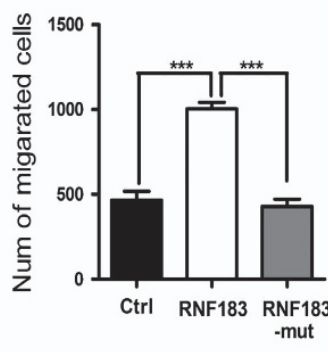

Figure 6 NF- $k$ B-IL-8 axis is indispensible for the oncogenic function of RNF183. (a and $\mathbf{b})$ NF- $k B$ inhibitor BAY11-7085 attenuates RNF183 overexpression induced migration (a) and invasion (b) of DLD-1 cells. (c) HCT116 cells were co-transfected with RNF183 plasmid and siRNA-targeting IL-8 as indicated. Recombinant IL-8 was also supplemented in the knockdown group to rescue the phenotype. The pictures of migrated cells (left) and quantification (right) were showed. (d) Luciferase activity of IL-8 promoter was evaluated in HCT116 cells with overexpression of control plasmid, wild-type RNF183 and truncated RNF183 without E3 ubiquitin ligase activity. (e-g) Effects of wild-type and truncated RNF183 on proliferation (e), colony formation (f) and migration (g) of HCT116 cells. Mean \pm S.D. $(n=3)$. Scale bar: $50 \mu \mathrm{m} .{ }^{* *} P<0.01,{ }^{* * *} P<0.001$

Cell viability assay. Cell growth was monitored by MTT assay. Treated or control cells at a density of $3 \times 10^{3}$ per well were grown in the 96-well plates in $0.1 \mathrm{ml}$ full medium at $37^{\circ} \mathrm{C}$ for $24 \mathrm{~h}$. Each indicated set had six duplicated wells, and MTT assays were repeated three times according to the manufacturer's protocol. ${ }^{29}$ Colony formation assays were in triplicate and conducted in six-well flat-bottom plates. The number of visible colonies was counted after staining with $5 \%$ crystal violet.

Migration and invasion assay. Cells were plated into the upper chamber with $8 \mu \mathrm{m}$ pore (BD Falcon, Franklin Lakes, NJ, USA) at a density of $1 \times 10^{5}$ per well. Chambers without or with Matrigel matrix for cells migration or invasion assays were inserted into matching 24-well plate containing MC5A 20\% FBS. Cells in the chamber were cultivated without fetal bovine serum. After $20 \mathrm{~h}$, migrated or invasive cells adhering to the lower surface of chamber were fixed and stained with $5 \%$ crystal violet and were counted in five fields of microscope.

Stable cell lines. For the overexpression experiments, HCT116 and DLD-1 cells were transfected with pcDNA4-myc/his-RNF183 or empty vector pcDNA4-myc/ his using Lipofectamine2000 (Invitrogen, Grand Island, NY). After 48 h, the RNF183 stable cells were selected by $300 \mu \mathrm{g} / \mathrm{ml}$ zeocin (Invitrogen) for additional 12 days. The culture medium was renewed every 3 days. For the knockdown experiments, RNF183 and control shRNA lentiviral vector was generated by GenePharma (GenePharma Corporation, Shanghai, China). Lentiviral vectors were transfected into HCT116 and DLD-1 cells prior to $3 \mu \mathrm{g} / \mathrm{ml}$ puromycin selection for 3 days. The stable RNF183 overexpression and knockdown cells were confirmed with qPCR and western blots.

Luciferase reporter assay. Dual-Luciferase Reporterassays (Promega) were performed according to the manufacturer's instruction. In brief, HCT116 cells were planted in 24-well plate $24 \mathrm{~h}$ prior to the transfection. After another $48 \mathrm{~h}$, Luciferase Assay Reagent II (LAR II) and Stop \& Glo Reagent sequentially, and the dual luciferase activity was measured. ${ }^{30,31}$

Xenograft tumor growth model. All animal experiments were conducted according to the institutional ethical and safe guidelines (Institutional Animal welfare and Ethics Committee, Sun Yat-sen university cancer center, Guangzhou, China). In total, 5-6-week-old female BALB/c nude mice were used in the experiments (SLAC laboratory animal Inc, Shanghai, China). WT-RNF183 stable overexpressed and Control DLD-1 xenograft model sets were established using $1 \times 10^{6}$ cells injection into the right flanks of subcutaneous tissue of mice. Tumor length and width and mice weight were measured every 2-3 days until the experiment was ended. The tumor volume was calculated based on the conventional formula: $V=\left(L \times W^{2}\right) / 2$, where $L$ and $W$ are the biggest and smallest diameters of the tumor.

Xenograft tumor metastasis model. For the pulmonary metastasis model, suspensions of stable cell lines were inoculated into the tail vein of nude mice $\left(1 \times 10^{6}\right.$ cells per mouse). For the intrahepatic metastasis model, mice were general anaesthetized by isoflurane, and the spleen was brought outside enterocoelia from $1 \mathrm{~cm}$ surgical incision on the left lower abdomen; the stable 
cell lines were injected into spleen and then, spleen was reset. After 8-10 weeks, the lungs and livers of mice were removed for pathological examination (H\&E staining). The number of metastasic nodules in lung and liver were counted under microscope.

IHC. Protein levels of RNF183 were detected by IHC assay with a peroxidase kit (DAKO, Carpinteria, CA, USA) as described previously. ${ }^{32}$ In brief, after routine deparaffinization, rehydration, and blocking with $0.3 \% \mathrm{H}_{2} \mathrm{O}_{2}$ and antigen retrieval, the slides were incubated overnight at $4{ }^{\circ} \mathrm{C}$ with anti-RNF183 antibody, followed by incubation with HRP-conjugated secondary antibody and visualized with the EnVision Detection Kit (DAKO). Then, the sections were counterstained with hematoxylin. RNF183 staining intensity and the percentage of corresponding positive area were evaluated by two pathologists who were blinded to clinical parameters. The RNF183 protein levels were presented as $\mathrm{H}$ score. ${ }^{32}$

ELISA and western blots. The secreted IL-8 was monitored by ELISA assays using human IL-8 ELISA kit (Proteintech, Wuhan, China). For western blots, total proteins were extracted from cells using cell lysis buffer containing protease inhibitor (Sigma-Aldrich, St. Louis, MO, USA). For western blots, samples were separated by $6-10 \%$ SDS-polyacrylamide gel electrophoresis, followed by transfer to PVDF membrane. After blocking in phosphate-buffered saline containing $5 \%$ bovine serum albumin and $0.1 \%$ Tween- 20 , the membrane was incubated with primary antibody at $4{ }^{\circ} \mathrm{C}$ overnight, followed by incubation with a peroxidase-linked secondary antibody (CalbioChem, Darmstadt, Germany) at room temperature for $1 \mathrm{~h}$. The signals were detected using Western blotting Luminol Reagent (Santa Cruz Inc.).

ChIP. ChIP Assay Kit was purchased from Beyotime (Shanghai, China). In brief, $3 \times 10^{6}$ cells with or without RNF183 transfection were cross-linked using $1 \%$ formaldehyde for $10 \mathrm{~min}$. DNA fragments were sheared to $200-1000 \mathrm{bp}$ by ultrasound deal on ice. The lysates were slow-whirling overnight at $4{ }^{\circ} \mathrm{C}$ with anti-P65 antibody or Rabbit lgG. Then the P65 bound DNA was precipitated and purified, and the enrichment of P65 on IL-8 promoter was examined by qPCR.

RNA extraction and RT-PCR. Total RNA was extracted using Trizo (Invitrogen, Carlsbad, CA, USA). The cDNA was synthesiszed from $1 \mu \mathrm{g}$ of total RNA using M-MLV reverse transcriptase (Promega). SYBR Green was purchased from BIO-RAD (Hercules, CA, USA) for quantitative RT-RCR. ${ }^{33}$ The primer sequence of target genes and reference gene GAPDH were listed in Supplementary Table 2.

Statistical analysis. Statistical analyses were completed using the SPSS version 16.0 (SPSS Inc., Chicago, IL, USA). ${ }^{34,35}$ Two-tailed $\chi^{2}$-tests were performed to assess significant associations between RNF183 levels and clinicopathological parameters. Survival analysis was carried out using the Kaplan-Meier method, and the log-rank test was used to compare the survival curves. The Cox proportional hazards model was used to calculate the univariate and multivariate hazards ratios for clinicopathological parameters and the RNF183 level with respect to OS and PFS. Other data were assessed with Student's two-tailed $t$-tests. $P<0.05$ was considered statistically significant. $P$-value $<0.05$ was considered statistically significant.

\section{Conflict of Interest}

The authors declare no conflict of interest.

Acknowledgements. This study was supported by the National Natural Science Foundation of China $(81672744,81472252)$, Science and Technology Project of Guangdong Province (2016A020217007), Guangdong Escophageal Cancer Institute Foundation (M201606) and Science and Technology Project of Guangzhou City (201707020039).

\section{Publisher's Note}

Springer Nature remains neutral with regard to jurisdictional claims in published maps and institutional affiliations.

1. Tenesa A, Dunlop MG. New insights into the aetiology of colorectal cancer from genome-wide association studies. Nat Rev Genet 2009; 10: 353-358.

2. Fearon ER. Molecular genetics of colorectal cancer. Annu Rev Pathol 2011; 6: 479-507.
3. Lakatos PL, Lakatos L. Risk for colorectal cancer in ulcerative colitis: changes, causes and management strategies. World J Gastroenterol 2008; 14: 3937-3947.

4. De Roock W, De Vriendt V, Normanno N, Ciardiello F, Tejpar S. KRAS, BRAF, PIK3CA, and PTEN mutation: implications for targeted therapies in metastatic colorectal cancer. Lancet Oncol 2011; 12: 594-603.

5. Normanno N, Tejpar S, Morgillo F, De Luca A, Van Cutsem E, Ciardiello F. Implications for KRAS status and EGFR-targeted therapies in metastatic CRC. Nat Rev Clin Oncol 2009; 6 : 519-527.

6. Corcoran RB, Ebi $\mathrm{H}$, Turke AB, Coffee EM, Nishino M, Cogdill AP et al. EGFR-mediated re-activation of MAPK signaling contributes to insensitivity of BRAF mutant colorectal cancers to RAF inhibition with vemurafenib. Cancer Discov 2012; 2: 227-235.

7. Nakamura $\mathrm{N}$. The role of the transmembrane RING finger proteins in cellular and organelle function. Membranes (Basel) 2011; 1: 354-393.

8. Ho SR, Mahanic CS, Lee YJ, Lin WC. RNF144A, an E3 ubiquitin ligase for DNA-PKcs, promotes apoptosis during DNA damage. Proc Natl Acad Sci USA 2014; 111: E2646-E2655.

9. Yang Y, Schmitz R, Mitala J, Whiting A, Xiao W, Ceribelli M et al. Essential role of the linear ubiquitin chain assembly complex in lymphoma revealed by rare germline polymorphisms. Cancer Discov 2014; 4: 480-493.

10. Giannakis M, Hodis E, Jasmine Mu X, Yamauchi M, Rosenbluh J, Cibulskis K et al. RNF43 is frequently mutated in colorectal and endometrial cancers. Nat Genet 2014; 46 : $1264-1266$.

11. Jiang X, Hao HX, Growney JD, Woolfenden S, Bottiglio C, Ng N et al. Inactivating mutations of RNF43 confer wnt dependency in pancreatic ductal adenocarcinoma. Proc Natl Acad Sci USA 2013; 110: 12649-12654.

12. Yu $Q$, Zhang $S$, Chao $K$, Feng $R$, Wang $H$, Li $M$ et al. E3 ubiquitin ligase RNF183 is a novel regulator in inflammatory bowel disease. J Crohns Colitis 2016; 10: $713-725$.

13. Jess T, Simonsen J, Jorgensen KT, Pedersen BV, Nielsen NM, Frisch M. Decreasing risk of colorectal cancer in patients with inflammatory bowel disease over 30 years. Gastroenterology 2012; 143: 375-381.

14. Beaugerie L, Svrcek M, Seksik P, Bouvier AM, Simon T, Allez M et al. Risk of colorectal high-grade dysplasia and cancer in a prospective observational cohort of patients with inflammatory bowel disease. Gastroenterology 2013; 145: 166-175.

15. Chapard $C$, Hohl D, Huber M. The role of the TRAF-interacting protein in proliferation and differentiation. Exp Dermatol 2012; 21: 321-326.

16. Chakraborty A, Diefenbacher ME, Mylona A, Kassel O, Behrens A. The E3 ubiquitin ligase Trim7 mediates C-Jun/AP-1 activation by RAS signaling. Nat Commun 2015; 6: 6782.

17. Hanahan D, Weinberg RA. Hallmarks of cancer: the next generation. Cell 2011; 144: 646-674.

18. Collins TS, Lee LF, Ting JP. Paclitaxel up-regulates interleukin-8 synthesis in human lung carcinoma through an NF-kappaB and AP-1 dependent mechanism. Cancer Immunol Immunother 2000; 49: 78-84.

19. Jayatilaka H, Tyle $P$, Chen JJ, Kwak M, Ju J, Kim HJ et al. Synergistic IL-6 and IL-8 paracrine signalling pathway infers a strategy to inhibit tumour cell migration. Nat Commun 2017; 8: 15584.

20. Grivennikov SI, Greten FR, Karin M. Immunity, inflammation, and cancer. Cell 2010; 140: 883-899.

21. Ammirante M, Luo JL, Grivennikov S, Nedospasov S, Karin M. B-cell-derived lymphotoxin promotes castration-resistant prostate cancer. Nature 2010; 464: 302-305.

22. Rubin DT, Huo D, Kinnucan JA, Sedrak MS, McCullom NE, Bunnag AP et al. Inflammation is an independent risk factor for colonic neoplasia in patients with ulcerative colitis: a case-control study. Clin Gastroenterol Hepatol 2013; 11: 1601-1608.

23. Atreya I, Neurath MF. Immune cells in colorectal cancer: prognostic relevance and therapeutic strategies. Expert Rev Anticancer Ther 2008; 8: 561-572.

24. Waldner MJ, Neurath MF. Cytokines in colitis associated cancer: potential drug targets? Inflamm Allergy Drug Targets 2008; 7: 187-194.

25. Sakamoto K, Maeda S, Hikiba Y, Nakagawa H, Hayakawa Y, Shibata W et al. Constitutive NF-kappaB activation in colorectal carcinoma plays a key role in angiogenesis, promoting tumor growth. Clin Cancer Res 2009; 15: 2248-2258.

26. Vlantis K, Wullaert A, Polykratis A, Kondylis V, Dannappel M, Schwarzer R et al. NEMO prevents RIP kinase 1-mediated epithelial cell death and chronic intestinal inflammation by NF-KB-dependent and -independent functions. Immunity 2016; 44 : 553-567.

27. Ning Y, Lenz HJ. Targeting IL-8 in colorectal cancer. Expert Opin Ther Targets 2012; 16: 491-497.

28. Jin WJ, Xu JM, Xu WL, Gu DH, Li PW. Diagnostic value of interleukin-8 in colorectal cancer: a case-control study and meta-analysis. World J Gastroenterol 2014; 20 : 16334-16342.

29. Shi W, Ye Z, Zhuang L, Li Y, Shuai W, Zuo Z et al. Olfactomedin 1 negatively regulates $\mathrm{NF}-\kappa \mathrm{B}$ signalling and suppresses the growth and metastasis of colorectal cancer cells. $J$ Pathol 2016; 240: 352-365.

30. Gao S, Wu J, Liu R, Li J, Song L, Teng Y et al. Interaction of NS2 with AIMP2 facilitates the switch from ubiquitination to SUMOylation of M1 in influenza A virus-infected cells. J Virol 2015; 89: 300-311. 
31. Chen S, Sheng C, Liu D, Yao C, Gao S, Song L et al. Enhancer of zeste homolog 2 is a negative regulator of mitochondria-mediated innate immune responses. J Immunol 2013; 191: 2614-2623.

32. Ding L, Chen S, Liu P, Pan Y, Zhong J, Regan KM et al. CBP loss cooperates with PTEN haploinsufficiency to drive prostate cancer: Implications for epigenetic therapy. Cancer Res 2014; 74: 2050-2061.

33. Wang $Q$, Jiang W, Chen Y, Liu P, Sheng C, Chen S et al. In vivo electroporation of minicircle DNA as a novel method of vaccine delivery to enhance HIV-1-specific immune responses. J Virol 2014; 88: 1924-1934.

34. Chen S, Zhao X, Tan J, Lu H, Qi Z, Huang Q et al. Structure-based identification of small molecule compounds targeting cell cyclophilin a with anti-HIV-1 activity. Eur $\mathrm{J}$ Pharmacol 2007; 565: 54-59.

35. Song L, Gao S, Jiang W, Chen S, Liu Y, Zhou L et al. Silencing suppressors: viral weapons for countering host cell defenses. Protein Cell 2011; 2: 273-281. cc) (i) Cell Death and Disease is an open-access journal published by Nature Publishing Group. This work is licensed under a Creative Commons Attribution 4.0 International License. The images or other third party material in this article are included in the article's Creative Commons license, unless indicated otherwise in the credit line; if the material is not included under the Creative Commons license, users will need to obtain permission from the license holder to reproduce the material. To view a copy of this license, visit http://creativecommons.org/licenses/by/4.0/

(C) The Author(s) 2017

Supplementary Information accompanies this paper on Cell Death and Disease website (http://www.nature.com/cddis) 